\title{
EVALUACIÓN DE ANTÍGENOS DE Fasciola hepatica POR TRES MÉTO- DOS INMUNOLÓGICOS
}

\section{ANTIGENS EVALUATION OF Fasciola hepatica FOR THREE IMMUNOLOGIC METHODS}

\author{
Erasmo Colona, Libertad Alzamora y Julia Castro
}

\section{RESUMEN}

Se evaluaron los antigenos de excreción-secreción (AES) de Fasciola hepatica en muestras de heces de ganado vacuno procedente de áreas endémicas de la Región Andrés Avelino Cáceres (Sierra Central), mediante los métodos inmunológicos de ELISA ("Fascidig"), hemaglutinación indirecta (HAl) y aglutinación en látex (AL). Los resultados obtenidos se correlacionaron con el método coproparasitológico de Dennis (CD). Se tomaron 106 muestras de heces de bovino en diferentes distritos, entre octubre y noviembre de 1995 (época lluviosa).

El método de AL no detectó los AES; mediante $\mathrm{HAl}$ la especificidad fue del $63,6 \%$ y la sensibilidad del $35 \%$ en relación al CD; por ELISA ("Fascidig") se obtuvo una especificidad del $68,2 \%$ y sensibilidad del $60 \%$ en relación al método CD y con respecto a $\mathrm{HAl}$ la especificidad fue $61,7 \%$ y la sensibilidad $50 \%$.

Se determinó una correlación significativa entre el método de ELISA ("Fascidig") y el CD, 10 que indica que un diagnóstico por ELISA permite conocer la enfermedad durante la fase prepatente o patente en relación al $C D$, que diagnostica la enfermedad durante la fase patente.

El diagnóstico de la infección o prevalencia por HAI, ELISA y CD fue $35,8 \%, 42,5 \%$ y $37,7 \%$ respectivamente. El índice encontrado corresponde a una zona de alta prevalencia de Fasciola hepatica.

El preserte estudio permitió evaluar tres métodos inmunológicos y determinar la eficiencia de ELISAFascidig como el método que posibilitaría el diagnóstico de la fasciolosis durante la fase prepatente y patente de la enfermedad por hallazgo de coproantigenos.

Palabras clave: Fasciola hepatica, Fascidig, Fascioliosis, Coproantigenos, Antigenos de excreción-secreción.

\section{ABSTRACT}

Excretion-secretion antigens (ESA) of Fasciola hepatica were evaluated in bovine feces samples from endemic area (Andrés Avelino Cáceres, Central Peruvian Andean). We employed ELISA (Fascidig), Indirect hemagglutination (IHA) and Latex agglutination (LA); the results were correlationated with Dennis coproparasitologic method (DC). A number of 106 bovine feces samples were obtained from diferent places of the Region between October and November 1995 (rain time).

AES was not detected for LA, the specificity for IHA was $63,6 \%$ and the sensibility $35 \%$ in relation to DC; the specificity for Elisa (Fascidig) was $68,2 \%$ and the sensibility was $60 \%$ with regard to DC; and in relation to IHA the specificity was $61,7 \%$ and the sensibility $50 \%$.

The correlation between ELISA (Fascidig) and DC was significative, it indicates that ELISA is the best method for the fasciolosis diagnostic in the prepatent and patent phases, while DC only is recommended for the patent phase diagnostic.

Diagnostic of infection or prevalence for IHA, ELISA and DC was $35,8 \%, 42,5 \%$ and $37,7 \%$ respectively. The results indicate that in the investigated area the prevalence of Fasciola hepatica is high.

"Laboratorio de Inmunoparasitologia y Epidemiología. Instituto de Investigación de Ciencias, Biológicas "Antonio Raimondi". UNMSM. Lima-Perú. 
Thanks to this investigation it was possible to evaluate three immunologic methods and to determine the performance of ELISA-Fascidig for the diagnostic of fasciolosis both prepatent and patent infection phases by means of the demonstration of coproantigens.

Keywords: Fasciola hepatica, Fascidig, Fasciolosis, Coproantigens, Excretion-secretion antigens.

\section{INTRODUCCIÓN}

La fasciolosis o distomatosis hepática es una parasitosis cosmopolita de importancia médico-veterinaria ocasiunada por Fasciola hepática, tremátode qui localizándose en los conductos biliares afecta a mamíferos silvestres, domésticos (ganado vacuno y ovino) y al hombre.

Esta parasitosis generalmente presenta dos fases: la fase prepatente o aguda y la fase patente. La fase prepatente es el período durante el cual las fasciolas inmaduras migran a través de la cavidad peritoneal, ingresan al hígado y luego al parénquima hepático; y permanecen en los conductos biliares hasta su madurez. En esta fase no se eliminan huevos del parásito en heces o fluido biliar.

La fase prepatente de la enfermedad puede diagnosticarse mediante exámenes coproparasitológicos. Aunque la intermitencia en la expulsión de los huevos no permite un diagnóstico exacto, si durante esta fase se realiza una ingesta constante de un pequeño número de metacercarias el organismo produce resistencia al parásito, lo que se evidencia con una marcada fibrosis y engrosamiento de los canalículos biliares.

La dificultad en diagnosticar el parasitismo por métodos coproparasitológicos e inmunológicos convencionales (detección de anticuerpos) ha llevado al estudio de los antígenos parasitarios en difercntes muestras biológicas (Castro et al., 1994).

Durante las fases prepatente y palente de la enfermedad no se hacen notorios los signos patognomónicos, por lo cual la detección de antigenos parasitarios es de importancia, ya que estos se relacionan con una infección reciente (parasitosis activa), lo que constitu- ye una ventaja corr respecto a la determinación de anticuerpos.

Debido al incremento de esta parasitosis, la que es favorecida por las condiciones ambientales y factores socioeconómicos de nuestro país, este trabajo tiene por objeto la evaluación de tres métodos inmunológicos (EL.ISA-Fascidig, Hemaglutinación Indirecta y Aglutinación en látex) que permitan diagnosticar la fasciolosis durante el período prepatente de la enfermedad, comparando la eficiencia de los mismos con el examen coproparasitulógico.

\section{MATERIALES Y MÉTODOS}

\section{Obtención de Fasciola hepatica}

Se obtuvieron fasciolas vivas de hígados parasitados de ovino y se utilizaron en la preparación del antigeno de excreción-secreción (AES).

\section{Colección de muestras de heces}

Se colectaron 106 muestras de heces de bovino provenientes de los distritos de $\mathrm{La}$ Oroya, San Lorenzo, Cullpa Alta, Chaquicocha, Matahuasi, Huantaro, Acostambo, Sapallanga, Chongos Alto y el entonces Instituto Peruano de Seguridad Social (hoy EsSalud) de Huancayo, ubicados en la Región Andrés Avelino Cáceres, durante los meses de octubre y noviembre de 1995 denominada épocal lluviosa por Leguía et al. (1988).

\section{Procesamiento de las muestras de heces}

Se pesó un gramo de heces de bovino. en viales de penicilina y se homogenizó 
con $3 \mathrm{mI}$, de agua desionizada ( $\mathrm{pH} 6,6$ ), se filtraron en gasa recogiéndose el filtrado en tubos. Los tubos se dejaron sedimentar durante toda la noche a $4^{\circ} \mathrm{C}$. El sobrenadante fue colectado y se procedió a la determinación de AES por ELISA y HAI.

\section{Preparación del antigeno de excreción- secreción empleado para la obtención de suero anti-AES}

Las fasciolas vivas de ovinos se colectaron en solución salina amoriguadora de fosfatos (SSAF) estéril pH 7,2, con $100 \mathrm{mg} /$ $\mathrm{mL}$ de estreptomicina y de ampicilina (solución de mantenimiento: SM), se lavaron 6 veces con SM, se seleccionaron fasciolas vivas en un matraz con SM y se incubaron a $37^{\circ} \mathrm{C}$ por 24 horas.

La solución se colectó a $4^{\circ} \mathrm{C}$ (en baño de hielo) y se centrífugó a $3500 \mathrm{rpm}$ por $45 \mathrm{mi}$ nutos, el sobrenadante fue colectado a $4^{\circ} \mathrm{C}$ y almacenado a $-20^{\circ} \mathrm{C}$ hasta su uso.

El dosaje de proteinas se realizó por el método de Biuret.

\section{Obtención del suero hiperiunmune anti-AES}

Se inmuni<ó un conejo albino con AES (0,25 mg, por dosis) por vía intramuscular empleando Coadyuvante Completo de Freund. El suero fue repartido en alicuotas que se almacenaron a $-20^{\circ} \mathrm{C}$ hasta su uso.

\section{Diagnóstico de Fasciolosis}

\subsection{Método coproparasitológico}

Se utilizó la técnica de concentración y cuantificación de Dennis (Dennis et al., 1954).

Cinco gramos de heces de bovino se diluyeron en $2 \mathrm{~mL}$ de agua potable y se filtraron en tamices metálicos con gasa. El filltrado se colocó en una copa de sedimentación y se diluyó con agua potable dejándose en reposo durante 10 minutos. El sobrenadante se eliminó y se agregó al sedimento agua potable hasta llenar la copa de sedimentación. Este proceso se repitió dos veces consecutivas con la finalidad de clarificar el sobrenadante. Al finalizar el tercer pasaje se obtuvieron 2 a $3 \mathrm{~mL}$ de sedimento, y se observó al microscopio.

\subsection{Métodos inmunológicos}

\section{a) Aglutinación en Látex (pasiva reversa)}

Se mezclaron $1,5 \mathrm{~mL}$ de látex estandarizado (Bacto látex $0,81 \mu$, DIFCO) con $1,5 \mathrm{~mL}$ de suero hiperinmune sin diluir y se incubó a temperatura ambiente durante 30 minutos con agitación suave cada 10 minutos (Alzamora et al., 1999). El látex sensibilizado con antiAES se empleó para detectar AES en las muestras de heces procesadas.

\section{b) Hemaglutinación indirecta (HAl-pasiva reversa)}

Se emplearon glóbulos rojos de carnero tanizados y sensibilizados (GRCS) con suero hiperinmune de conejo anti-AES. La mezcla se incubó en baño María a $37^{\circ} \mathrm{C}$ durante 30 minutos. Los GRCS se centrifugaron a 1500 rpm durante 5 minutos, el sobrenadante se eliminó y las células se lavaron 2 veces usando $\mathrm{SSAF}$ con $1 \%$ de suero normal descomplementado de conejo (SNC); a 1500 rpm por 5 y 10 minutos. Del paquete obtenido se preparó una suspensión en SSAF-SNC 1\%.

La determinación del AES se realizó mezclando $40 \mu \mathrm{L}$ del sobrenadante de la muestra de heces y $40 \mu \mathrm{L}$ de GRCS. Las placas se dejaron en reposo a $4^{\circ} \mathrm{C}$ durante 18 horas.

\section{c) ELISA (Tipo Sandwich)}

Se empleó el kit diagnóstico "Fascidig" elaborado por el Instituto Pedro Kouri de La Habana-Cuba. Este método, que se aplicó según la técnica descrita por Espino et al.(1992 y 1994), se basa en la detección de antígenos en heces (coproantigenos) usando el anticuerpo monoclonal ES-78 de subclase IgG $2 \mathrm{a}$. 
Tabla 1. Detección de antigenos de excreción-secreción (coproantigenos) de Fasciola hepatica en heces de bovino mediante HAI, ELISA y coproparasitológico de Dennos. Las muestras se colectaron en los meses de Octubre y Noviembre de 1995.

\begin{tabular}{|c|c|c|c|c|}
\hline MUESTRA & PROCEDENCIA & HAI & ELISA & DENNIS \\
\hline $\begin{array}{l}1 \\
2\end{array}$ & LA OROYA & $\begin{array}{l}+ \\
- \\
\end{array}$ & $\begin{array}{l}+ \\
+ \\
\end{array}$ & $\begin{array}{l}+ \\
+\end{array}$ \\
\hline 3 & \multirow{5}{*}{ SAN LORENZO } & - & + & + \\
\hline 4 & & + & + & - \\
\hline 5 & & + & + & - \\
\hline 6 & & - & + & - \\
\hline 7 & & + & + & - \\
\hline 8 & \multirow{7}{*}{ CULLPA ALTA } & - & + & + \\
\hline 9 & & - & - & - \\
\hline 10 & & - & - & + \\
\hline 11 & & + & - & + \\
\hline 12 & & - & + & - \\
\hline 13 & & + & + & + \\
\hline 14 & & - & - & - \\
\hline 15 & \multirow{32}{*}{ CHAQUICOCHA } & - & - & - \\
\hline 16 & & + & + & + \\
\hline 17 & & - & + & + \\
\hline 18 & & + & + & - \\
\hline 19 & & - & - & - \\
\hline 20 & & . & + & - \\
\hline 21 & & - & + & - \\
\hline 22 & & + & + & + \\
\hline 23 & & + & - & - \\
\hline 24 & & + & - & - \\
\hline 25 & & - & - & + \\
\hline 26 & & - & - & - \\
\hline 27 & & - & - & - \\
\hline 28 & & - & - & - \\
\hline 29 & & + & - & - \\
\hline 30 & & - & - & . \\
\hline 31 & & - & + & - \\
\hline 32 & & - & + & - \\
\hline 33 & & - & + & - \\
\hline 34 & & - & + & - \\
\hline 35 & & - & $=$ & - \\
\hline 36 & & + & - & - \\
\hline 37 & & - & - & - \\
\hline 38 & & - & $=$ & - \\
\hline 39 & & + & - & . \\
\hline 40 & & - & - & - \\
\hline 41 & & - & - & - \\
\hline 42 & & + & + & + \\
\hline 43 & & + & - & - \\
\hline 44 & & - & $=$ & + \\
\hline 45 & & - & + & - \\
\hline 46 & & - & + & + \\
\hline 47 & \multirow{3}{*}{ MATAHUASI } & - & $=$ & - \\
\hline 48 & & + & + & + \\
\hline 49 & & - & + & + \\
\hline 50 & \multirow{3}{*}{ HLIANTARO } & + & + & - \\
\hline 51 & & - & + & + \\
\hline 52 & & - & + & + \\
\hline
\end{tabular}

\begin{tabular}{|c|c|c|c|c|}
\hline MUESTRA & PROCEDENCIA & HAI & ELISA & DENVIS \\
\hline$\overline{53}$ & \multirow{13}{*}{ ACOSTAMBO } & - & + & - \\
\hline 54 & & - & + & + \\
\hline 55 & & - & + & + \\
\hline 56 & & - & + & + \\
\hline 57 & & - & - & + \\
\hline 58 & & - & - & + \\
\hline 59 & & - & - & - \\
\hline 60 & & - & - & + \\
\hline 61 & & - & - & + \\
\hline 62 & & - & - & + \\
\hline 63 & & + & - & - \\
\hline 64 & & - & - & + \\
\hline 65 & & - & - & + \\
\hline 66 & \multirow{11}{*}{ SAPALLANGA } & - & - & - \\
\hline 67 & & t. & - & - \\
\hline 68 & & + & - & - \\
\hline 69 & & - & - & - \\
\hline 70 & & - & - & - \\
\hline 71 & & + & - & - \\
\hline 72 & & - & . & - \\
\hline 73 & & - & - & - \\
\hline 74 & & - & - & + \\
\hline 75 & & - & - & - \\
\hline 76 & & - & - & - \\
\hline 77 & \multirow{16}{*}{$\begin{array}{c}\text { CHON:UOS } \\
\text { ALTO }\end{array}$} & - & - & - \\
\hline 78 & & - & - & - \\
\hline 79 & & + & . & - \\
\hline 80 & & + & - & + \\
\hline 81 & & - & - & - \\
\hline 82 & & + & - & + \\
\hline 83 & & + & . & - \\
\hline 84 & & + & - & - \\
\hline 85 & & - & - & - \\
\hline 86 & & - & - & - \\
\hline 87 & & - & - & + \\
\hline 88 & & + & - & - \\
\hline 89 & & - & - & - \\
\hline 90 & & + & . & - \\
\hline 91 & & - & - & - \\
\hline 92 & & - & - & - \\
\hline 93 & \multirow{14}{*}{$\begin{array}{c}\text { IPSS- } \\
\text { HUANCAYO }\end{array}$} & + & - & + \\
\hline 94 & & - & + & + \\
\hline 95 & & - & + & + \\
\hline 96 & & + & + & $\div$ \\
\hline 97 & & + & $\therefore$ & + \\
\hline 98 & & + & + & - \\
\hline 99 & & + & + & + \\
\hline 100 & & - & + & - \\
\hline 101 & & + & + & - \\
\hline 102 & & - & + & + \\
\hline 103 & & + & + & - \\
\hline 104 & & + & + & - \\
\hline 105 & & - & + & - \\
\hline 106 & & + & + & + \\
\hline \multicolumn{2}{|c|}{ TOTAL } & ||$A \mid$ & EIISA & DENNIS \\
\hline \multicolumn{2}{|c|}{ MUESTRAS POSITIVAS } & 38 & 45 & $40^{*}$ \\
\hline
\end{tabular}

* 1 -5 huevos/gramo de neces

Las muestras se colectaron en los meses de octubre y noviembre de 1995. 
Tabia 2. Determinación de parasitosis por F hepatica segün el lugar de procedencia.

\begin{tabular}{rccc}
\hline & & & \\
PROCEDENCIA & & & \\
(Región Andrés & & & \\
A. Cáceres) & HAI & ELISA & DENNIS \\
\cline { 2 - 4 } & & & 2 \\
\hline La Oroya & 1 & 5 & 1 \\
San Lorenzo & 3 & 3 & 4 \\
Cullpa Alta & 2 & 13 & 7 \\
Chaquicocha & 10 & 2 & 2 \\
Matahuasi & 1 & 3 & 2 \\
Huantaro & 1 & 4 & 10 \\
Acostambo & 1 & 0 & 1 \\
Sapallanga & 3 & 0 & 3 \\
Chongos Alto & 7 & 13 & 8 \\
IPSS-Huancayo & 9 & 45 & 40 \\
Total & 38 & &
\end{tabular}

De cada muestra de heces se extrajeron 100 $\mu \mathrm{L}$, se colocaron en los pocillos de la placa de ELISA, y se incubaron dos horas a temperatura ambiente en cámara húmeda. Se lavaron 6 veces con el buffer de lavado del kit. Luego, a cada pocillo se agregaron $100 \mu \mathrm{L}$ del conjugado y se incubó durante 2 horas a temperatura ambiente en cámara húmeda. Los pocillos se lavaron 6 veces con el buffer de lavado y se añadió a cada pocillo $100 \mu \mathrm{L}$ de sustrato ortofenilendiamina (OPD) y posteriormente peróxido de hidrógeno al $30 \%$.

La placa se incubó por 30 minutos a temperatura ambiente, en oscuridad, y se procedió a la lectura.

\section{Procesamiento y análisis estadistico de los resultados}

En pruebas diagnósticas en las que se manejan variables categóricas dicotómicas $(+,-)$ la validez se conceptualiza en los términos de sensibilidad y especificidad. Para determinar la especificidad y la sensibilidad se emplearon las tablas de contingencia $2 \times 2$.

El valor predictivo positivo $\mathrm{Vp}(+)$ es definido como la proporción de sujetos con resul- tados positivos para la prueba y que realmente son positivos.

El valor predictivo negativo $\mathrm{Vp}(-)$ es definido como la proporción de sujetos con resultados negativos para la prueba y que reaimente son negativos.

Para determinar si existe correlación significativa entre los resultados de los 3 métodos inmunológicos y el examen coproparasitológico, se aplicó el método estadístico del Chi cuadrado.

\section{RESULTADOS}

I. Detección de AES en muestras de heces de ganado vacuno por los métodos de Aglutinación en Látex (AL), Hemaglutinación Indirecta (HAI) y ELISA (Fascidig)

Los resultados obtenidos en las pruebas de ELISA, HAI y CD por cada caso y distrito, se presentan en las tablas 1 y 2 . El método de aglutinación en látex no detectó el AES.

Mediante el método de HAI la especificidad fue del $63,6 \%$ y la sensibilidad del $35 \%$ en relación al coproparasitológico. Los valores predictivos positivos y negativos fueron $34,2 \%$ y $61,8 \%$ respectivamente (Tabla 3 ). 
Tabla 3. Especificidad y serisibilidad del método Hemaglutinación indirecta (HAI) con respecto al coproparasitológico de Dennis para coproantígenos de Fasciola hepatica en bovinos.

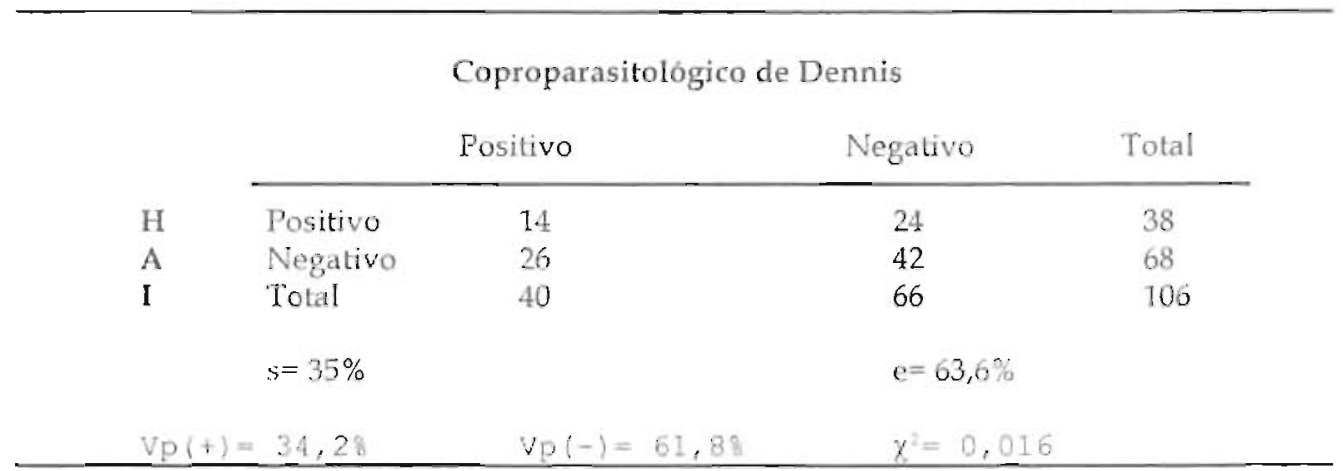

El método de ELISA ("Fascidig") mostró una especificidad del $68,2 \%$ y sensibilidad del $60 \%$ en relación al método coproparasitológico. Los valores predictivos positivos y negativos fucron del $53,3 \%$ y $73,8 \%$ respectivamente (Tabla 4 ).

El método ELISA mostró con respecto a HAI una especificidad de: $61,7 \%$, sensibilidad $50 \%$ y valores predictivos positivos y negativos del $42,2 \%$ y $68,9 \%$ respectivamente (Tabla 5)

II. Detección de huevos de Fasciola hepatica en muestras de heces por el método CD.

En cuarenta muestras, se determinaron de I a 5 huevos por gramo de heces.
III. Comparación de los métodos de Hemaglutinación Indirecta (HAI) y ELISA ("Fascidig") con el método coproparasitológico de Dennis (CD)

Con la finalidad de determinar la correlación entre los 3 métodos inmunológicos y el CD se obturo el Chi cuadrado con un nivel de significación estadistica del $10 \%$ que corresponde a un valor de 2,71.

La HAI con respecto al CD mostró un valor de 0,016, que no es significativo (menor que 2,71), mientras que ELISA en relación al CD obtuvo un valor de 8,19 , que si es significativo (mayor que 2,71).

Cuando se correlacionaron ELISA y HAI se encontró un valor de 1,45 que no es significativo (Tabla 5).

Tabla 4. Especificidad y sensibilidad del métoda ELISA (Fascidig) con respecto al coproparasitológico de Dennis para coproantigenos de Fasciola hepatica en bovinos.

\begin{tabular}{|c|c|c|c|c|c|}
\hline & & \multicolumn{3}{|c|}{ Coproparasitologgico de Dennis. } & \multirow[b]{2}{*}{ Total: } \\
\hline & Positivo & \multicolumn{2}{|c|}{ Negativo } & \\
\hline 1 & Positivo & 24 & & $2 \mathrm{I}$ & 45 \\
\hline S & Negativo & 16 & & 45 & Bi \\
\hline \multirow[t]{2}{*}{ A } & Total & 40 & & 00 & 100 \\
\hline & $s=60 \%$ & & & & \\
\hline \multicolumn{2}{|c|}{$V p(t)=53,3 \%$} & & $V p(-)=73,8 \%$ & & \\
\hline
\end{tabular}


Tabla 5. Especificidad y sensibilidad del método ELISA (Fascidig) con respecto al de Hemaglutinación Indirecta (Pasiva Reversa) para coproantigenos de Fasciola hepatica en bovinos.

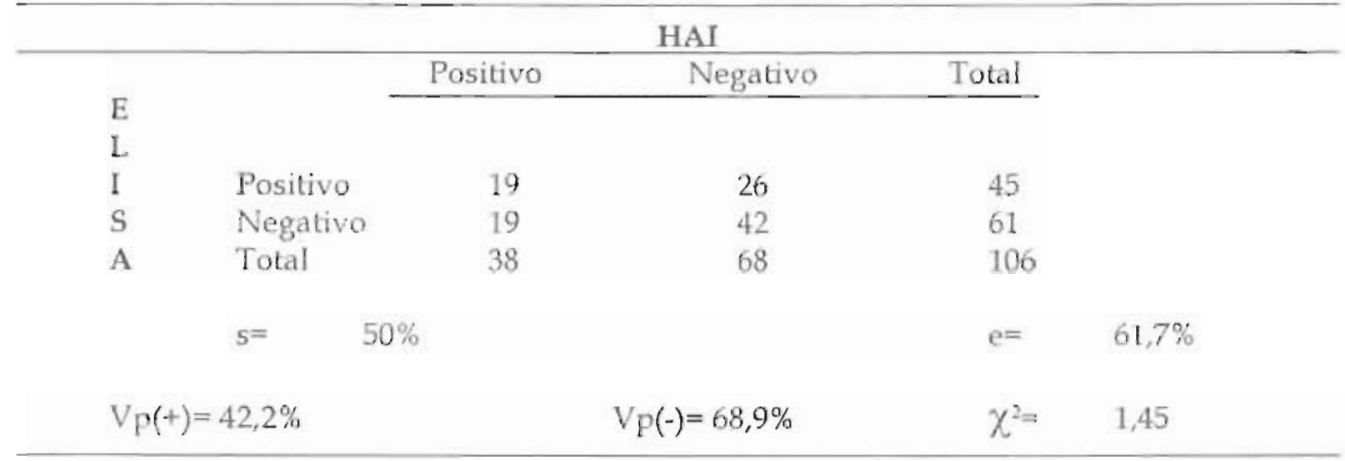

IV. Detección del estado de infección con F. hepatica (casos positivos) según el lugar de procedencia.

En las tablas I y 2 se describe la incidencia en los distintos lugares de procedencia.

Los Iugares de mayor incidencia, según HAI y ELISA fueron Chaquicocha e IPSSHuancayo; mientras que por el CD fueron Acostambo e IPSS-Huancayo, Los de menor incidencia según HAI fueron la Oroya, Matahuasi, Huantaro y Acostambo; por Elisa Sapallanga y Chongos Altos y por el CD San Lorenzo y Sapallanga.

Se mostró una similar incidencia en los lugares de la Oroya y Matahuasi con los métodos de ELISA y CD.

V. Evaluación del estado de infección por los métodos inmunológicos de Hemaglutinación Indirecta y ELISA (Fascidig) y el coproparasitológico de Dennis.

Por medio de HAl se determinaron un total de 38 casos positivos lo que corresponde al $35,8 \%$ del total de casos evaluados (Tabla 5 ).

EL método de ELISA detectó 45 casos positivos, lo que equivale al. $42,5 \%$ del total de casos evaluados. El mayor número de muestras positivas fueron determinadas por este método. El CD determinó una prevalencia deł $37,7 \%$ (Figura 1)
Se presentaron 9 casos negativos a CD y positivos tanto para HAI como para. ELISA. Esto pondria en evidencia la fase prepatente o aguda de la enfermedad en la que la presencia de huevos del parásito es nula.

Los casos positivos por los tres métodos se relacionarian con la fase patente de la enfermedad. Los casos positivos se incrementaron en un $19,8 \%$ y $22,6 \%$ al relacionar el método de ELISA con el CD respectivamente.

\section{DISCUSIÓN}

Los métodos inmunológicos convencionales y coproparasitológicos han sido lo suficientemente eficaces en el diagnóstico de las infecciones activas por parásitos; la alternativa para la detección se ha basado en la presencia de antígenos parasitarios en diversas muestras biológicas (Deplazes, et al. 1991; Espino, et al. 1994). En el caso particular de la fasciolosis, la fase prepatente se caracteriza por la ausencia de huevos del parásito y la fase patente por la intermitencia en la expulsión de los mismos (Levine et al., 1980; Chenm y Mott 1990).

La detección de antigenos parasitarios en muestras fecales (coproantígenos) debido a su fácil obtención, månejo y transporte resulta de gran ayuda para un diagnóstico certero. 


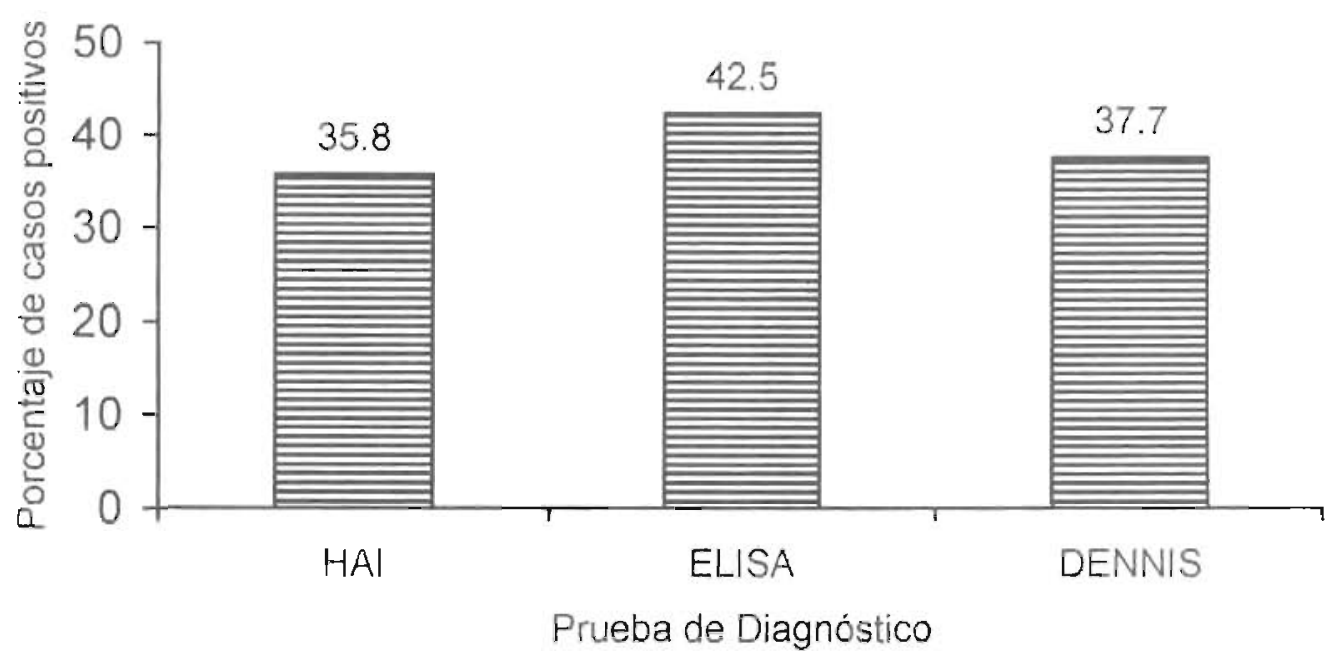

Figura 1. Resultados comparativos entre los métodos inmunológicos y el Dennis para el diagnóstico de la fascioliosis bovina (Región A.A. Cáceres - 1995)

Con relación a la hemaglutinación indirecta (pasiva reversa) se mostró una especificidad de $63,6 \%$ y una sensibilidad del $35 \%$, datos determinados tomando como método de referencia al coproparasitológico.

No hay reportes en los que se detecten antigenos en suero o heces por hemaglutinación indirecta que permitan comparar los resulados obtenidos en el presente estudio.

Sin embargo Knobloch (1985), evaluando anticuerpos en sueros de humanos con fasciolosis crónica, determinó por hemaglutinación indirecta una sensibilidad del $56 \%$, mientras que Gorman et al., en 1990 encontraron en sueros de ovinos una sensibilidad del $55,4 \%$.

De acuerdo a los resultados, el método HAI no es muy sensible (35\%) respecto al CD y ELISA; sin embargo, para la determinación de la fase prepatente mostró mayor eficiencia que el CD (Tabla 2), con casos negativos por CD pero coincidentemente positivos por ELISA y HAI. La baja sensibilidad podria deberse a que otras proteinas del suero hiperinmune utilizado interfieren en la prueba al unirse al glóbulo rojo de carnero tanizado, evitando una sensibilización uniforme con los anticuerpos anti-AES, se podría mejorar el método de HAl-pasiva reversa, ya que constituiría una alternativa económica para el diagnóstico de esta parasitosis.

Mediante la prueba de ELISA ("Fascidig") la especificidad y sensibilidad que se obtuvo con respecto al coproparasitológico de Dennis fue del $68,2 \%$ y $60 \%$ respectivamente. Estos datos son menores que los reportados por Castro et al., en 1994, los cuales utilizando el kit "Fascidig" para coproantigenos encontraron una especificidad del $99 \%$ y sensibilidad de $95,3 \%$. La diferencia de resultados podría tener relación con la baja densidad parasitaria encontrada, a diferencia de to reportado por Castra (1993), quien halló una elevada carga parasitaria por el método de Dennis (26 a 156 huevos/g de heces).

El método de ELISA en relación con HAI demostró una especificidad del $61,7 \%$ y sensibilidad del $50 \%$. No existen referencias que permitan discutir los datos encontrados. 
Se determinó una correlación significativa entre el "Fascidig" y el método de Dennis (Tabla 3), lo que indica que se puede realizar el diagnóstico de la enfermedad durante la fase prepatente o patente; cabe recalcar que el método de Dennis sólo permite el diagnóstico de la enfermedad durante la fase patente. Esta correlación se compara a lo reportado por Castro (1993) que encontró una relación significativa entre ambos métodos.

Con respecto a la incidencia, el mayor número de casos coincidentemente según $\mathrm{HAI}$ y ELISA fueron Chaquicocha e IPSSHuancayo mientras que por CD fueron Acostambo e IPSS-Huancayo (Tabla 2), datos que no se relacionan con los obtenidos por Castro y Colona (1996), quienes determinaron el mayor número casos por ELISA en las comunidades de Chongos Alto y Matahuasi. Estos resultados indican variación de la parasitosis en relación al tiempo.

El menor número de muestras colectadas explicaría la diferencia demostrada con el reporte de Castro y Colona (1996); sin embargo, en Chongos Alto aunque el número de muestras fue mayor, no se detectaron casos positivos por el método de ELISA probablemente debido a una baja densidad parasitaria por individuo, ya que no se puede descartar totalmente el parasitismo, además de las investigaciones realizadas por Castro (1993), que demostró en animales de matadero la existencia de una correlación estadísticamente significativa entre la intensidad de la infección, la excreción de huevos y la concentración de antígenos en heces.

El estado de infección o prevalencia determinado por HAI fue del $35,8 \%$ mientras que el obtenido por ELISA y el método de Dennis fue del $42,5 \%$ y $37,7 \%$ respectivamente (Figura 1). E! índice encontrado corresponde a una zona de alta prevalencia de fasciolosis de acuerdo a los datos reportados por Leguía et al, 1988.

Estos investigadores clasificaron la prevalencia de distomatosis por áreas, y se consi- dera el departamento de Junin (Región A. A. Cáceres) como un lugar de alta prevalencia.

Los resultados obtenidos en el presente estudio $(42,5 \%)$ y en el realizado por Castro y Colona en 1996 (74,02\%) también dernuestran que la zona investigada es de alta prevalencia para Fasciola hepatica.

El presente estudio permitió evaluar ties métodos inmunológicos ( $\mathrm{HAl}$, ELISA y CD) y determinar la eficiencia de ELISA"Fascidig" para diagnosticar la fasciolosis durante la fase prepatente y patente de la enfermedad por hallazgo de coproantígenos.

\section{AGRADECIMIENTOS}

Al Dr. Jorge Ruiz Salomé, Jefe de la Oficina Veterinaria del Frigorifico Moderna S. A., y al Sr. Edinson Villanueva por la ayuda prestada para la obtención del material biológico.

A la Bach. María Maysundo Reyes por su apoyo en la colección y transporte del material biológico.

\section{LITERATURA CITADA}

Alzamora, L.; R. Torres and E. Colona 1999. A rapid test for detection of antibodies against measles virus. Libro de Resúmenes del V Congreso Latinoamericano de Inmunologia. Punta del Este-Unguay. Pág. 120.

Castro, J. 1993. Evaluación del estado de infección por Fasciola hepatica en tres unidades pecuarias de la ciudad de La IJabana, utilizando el kit diagnóstico FASCIDIG (Tesis de Maestría). Instituio de Investigación "Pedro Kouri" La Habana-Cuba.

Castro, J.; B. Duménigo y A. Espino. 1994. Detección de coproantigenos para evaluar la infección activa por Fasciola hepatica en ganado bovino. Parasitología al Día 18:33-38.

Castro, 1. y E. Colona. 1996. Diagnóstico de Fasciola hepalica a través de la captura de coproantigenos. Libro de Resủmenes de la V Reunión Cientifica ICBAR Lima-Perú.

Chenm, M. K. and K.. Mott. 1990. Progress in assessment of morbidity due to Fasciola hepatica infection: A review of recent literature. Tropical Diseases Bulletin 87:1-38. 
Dennis, W; W. Stone and L. Swanson. 1954. A new Laboratory and field diagnostic test for lluke ova in feces. Journal American Veterinary Medical Association 124: 47-50.

Deplazes, P.; J. Eckeri; Z. S. Pawlowski; L. Machowska and B. Gottstein. 1991. An enzyme-linked immunosorbent assay for diagnostic detection of Taenia saginata copro-antigens in humans. Transactions of the Royal Society of Tropical Medicine and Hygiene 85:391-396.

Espino, A. M.; J. C. Millan and C. M. Finlay. 1992. Detection of antibodies and circulating excretory-secretory antigens for assessing cure in patients with fascioliasis. Transactions of the Royal Society of Tropical Medicine and Hygiene 86:000-000.

Espino, A. M. and C. Finlay. 1994. Sandwich Enzymelinked immunosorbent assay for detection of excretory-secretory antigens in humans with fascioliasis. Journal of Clinical Microbiology 32(1): 190-193.
Gorman T.; J. Wenzel; M. Lorca; L. Ibarra; B. San Martin y H. Aicaino. 1990. Pruebas de inmunoprecipitación y hemoaglutinacicill i:directa en el diagnóstico de la fascioliastis ovina. Parasitologia al Día 14: 51-56.

Knobloch J. 1985. Human fascioliasis in Cajanarca/ Perú. Il Humoral ancibody response and antigenaemia. Tropical Medicine and Parasitology 36(2): 91-93.

Leguia G.; H. Álvarez; F. Náquira y M. Beltrún. 1988. Distomatosis hepática en el Perú. Anales dét Seminario Nacional de Zoonosis y Lnfermedades de Transmisión Almentaria. Pides. 96106.

Levine. D. M.; G. V. Hillyer and S. I. Flores. 1980. Comparison of counterelectrophor sis, the enzyme-linked immunosorbent assay, and Kato fecal examination for the diagno:its of fascioliasis in infected mice and rabbits. American Journal of Tropical Medicine and Hygiene 29(4): 602-608. 